\title{
Vector-like quarks at the origin of light quark masses and mixing
}

\author{
Francisco J. Botella ${ }^{1, \mathrm{a}}$, G. C. Branco ${ }^{2, \mathrm{~b}}$, Miguel Nebot ${ }^{2, \mathrm{c}}$, M. N. Rebelo ${ }^{2, \mathrm{~d}}$, J. I. Silva-Marcos ${ }^{2, \mathrm{e}}$ \\ ${ }^{1}$ Departament de Física Teòrica and IFIC, Universitat de València-CSIC, 46100 Burjassot, Spain \\ 2 Departamento de Física and Centro de Física Teórica de Partículas (CFTP), Instituto Superior Técnico (IST), Universidade de Lisboa (UL), \\ Av. Rovisco Pais, 1049-001 Lisbon, Portugal
}

Received: 16 March 2017 / Accepted: 17 May 2017 / Published online: 17 June 2017

(C) The Author(s) 2017. This article is an open access publication

\begin{abstract}
We show how a novel fine-tuning problem present in the Standard Model can be solved through the introduction of a $Z_{6}$ flavour symmetry, together with three $Q=-1 / 3$ quarks, three $Q=2 / 3$ quarks, as well as a complex singlet scalar. The $Z_{6}$ symmetry is extended to the additional fields and it is an exact symmetry of the Lagrangian, only softly broken in the scalar potential, in order to avoid the domain-wall problem. Specific examples are given and a phenomenological analysis of the main features of the model is presented. It is shown that even for vector-like quarks with masses accessible at the LHC, one can have realistic quark masses and mixing, while respecting the strict constraints on processes arising from flavour changing neutral currents. The vector-like quark decay channels are also described.
\end{abstract}

\section{Introduction}

In the framework of the Standard Model (SM), the BroutEnglert-Higgs mechanism is responsible not only for the breaking of the gauge symmetry but also for the generation of fermion masses, through the Yukawa interactions of the scalar doublet with quarks and leptons. Understanding the observed pattern of fermion masses and mixing remains a fundamental open question in Particle Physics. On the experimental side, there has been great progress and at present the moduli of the Cabibbo-Kobayashi-Maskawa (CKM) matrix, $V_{\mathrm{CKM}}$, are reasonably well known [1-3] with clear evidence that the mixing matrix is non-trivially complex, even if one allows for the presence of New Physics (NP) beyond the SM [4-6]. The discovery of the Higgs particle at LHC [7,8] ren-

\footnotetext{
a e-mail: francisco.j.botella@uv.es

be-mail: gbranco@tecnico.ulisboa.pt

ce-mail: nebot@cftp.tecnico.ulisboa.pt

de-mail: rebelo@tecnico.ulisboa.pt

ee-mail: juca@cftp.tecnico.ulisboa.pt
}

ders specially important the measurement of the Higgs couplings to quarks and charged leptons. The strength of these couplings is fixed within the SM, but at present we have poor knowledge of their actual value.

Recently, it has been pointed out [9] that there is a novel fine-tuning problem in the SM. Contrary to conventional wisdom, in the SM without extra flavour symmetries, quark mixing is naturally large, in spite of the large quark mass hierarchy. In fact, even in the extreme chiral limit, where only the third family acquires mass, mixing is meaningful, and in general it is of order one. It is possible to solve this finetuning problem through the introduction of a simple flavour symmetry which leads to $V_{\mathrm{CKM}}=\mathbb{1}$. The challenge is then to achieve quark mixing and masses for the first two generations.

In this paper we face this challenge and put forward a framework where a flavour symmetry leads to $V_{\mathrm{CKM}}=\mathbb{1}$ in leading order, with light quark masses and mixing being generated by the presence of vector-like quarks (V1Q). We introduce three up-type VlQ and three down-type VlQ and derive effective mass squared Hermitian mass matrices for the standard quarks. In this framework one finds a natural explanation why $\left|V_{13}\right|^{2}+\left|V_{23}\right|^{2}$ is very small, while allowing for an adequate Cabibbo mixing. In this model there are Z-mediated and Higgs-mediated Flavour Changing Neutral Currents (FCNC) which are naturally suppressed. The VlQs can have masses of the order of one $\mathrm{TeV}$ which are at the reach of the second run of LHC [10-19]. At this stage, it is worth emphasising that VlQs have been extensively studied in the literature [20-48] and arise in a variety of frameworks, including E6 GUTS, models with extra dimensions, models providing solutions to the strong $\mathrm{CP}$ problem without axions, etc. It has also been pointed out [49] that non-supersymmetric extensions of the SM with VlQs can achieve unification of gauge couplings.

The paper is organised as follows: in the next section we briefly review a novel fine-tuning problem of the Standard 
Model and illustrate how it can be solved through the addition of a flavour symmetry. In Sect. 3, we show how a realistic quark mass spectrum and pattern of mixing can be generated through the introduction of vector-like quarks and a complex scalar singlet. In Sect. 4 we give specific examples examining the constraints arising from various FCNC processes and describing the vector-like quark decay channels. Finally, our conclusions are presented in Sect. 5.

\section{Hierarchy and alignment through a flavour symmetry}

\subsection{A novel fine-tuning problem in the standard model}

Recently, it has been pointed out [9] that in the SM there is a novel fine-tuning problem which stems from the fact that the natural value of $\left|V_{13}\right|^{2}+\left|V_{23}\right|^{2}$ is of order one in the $\mathrm{SM}$, to be compared to the experimental value of $1.6 \times 10^{-3}$. In order to obtain this result, one can examine the extreme chiral limit where only the third family of quarks has mass while all other quarks are massless. In this limit, the general quark mass matrices can be written

$M_{d}=U_{L}^{d^{\dagger}} \operatorname{diag}\left(0,0, m_{b}\right) U_{R}^{d}$,

$M_{u}=U_{L}^{u^{\dagger}} \operatorname{diag}\left(0,0, m_{t}\right) U_{R}^{u}$

where $U_{L, R}^{d, u}$ are arbitrary unitary matrices. The quark mixing matrix is then $V^{0}=U_{L}^{u \dagger} U_{L}^{d}$. Using the freedom to redefine the quark fields of the massless quarks through two-by-two unitary matrices one can show that the quark mixing can be described by an orthogonal two-by-two rotation connecting only the $(c, t)$ and $(s, b)$ quarks. This angle is arbitrary and expected to be of order one.

\subsection{A flavour symmetry leading to small mixing}

Let us consider the $\mathrm{SM}$ and introduce the following $Z_{6}$ symmetry:

$$
\begin{aligned}
& Q_{L 1}^{0} \rightarrow e^{i \tau} Q_{L 1}^{0} \quad Q_{L 2}^{0} \rightarrow e^{-2 i \tau} Q_{L 2}^{0} \quad Q_{L 3}^{0} \rightarrow e^{-i \tau} Q_{L 3}^{0} \\
& d_{R 1}^{0} \rightarrow e^{-i \tau} d_{R 1}^{0} \quad d_{R 2}^{0} \rightarrow e^{-i \tau} d_{R 2}^{0} \quad d_{R 3}^{0} \rightarrow e^{-2 i \tau} d_{R 3}^{0} \\
& u_{R 1}^{0} \rightarrow e^{i \tau} u_{R 1}^{0} \quad u_{R 2}^{0} \rightarrow e^{i \tau} u_{R 3}^{0} \quad u_{R 3}^{0} \rightarrow u_{R 3}^{0} \\
& \Phi \rightarrow e^{i \tau} \Phi ; \quad \tau=\frac{2 \pi}{6}
\end{aligned}
$$

where the $Q_{L j}^{0}$ are left-handed quark doublets, $d_{R j}^{0}$ and $u_{R j}^{0}$ are right-handed quark singlets and $\Phi$ denotes the Higgs doublet. The Yukawa interactions are given by

$$
\mathcal{L}_{\mathrm{Y}}=\left[-\bar{Q}_{L i}^{0} \Phi Y_{d} d_{R j}^{0}-\bar{Q}_{L i}^{0} \tilde{\Phi} Y_{u} u_{R j}^{0}\right]+\text { h.c. }
$$

and this symmetry leads to the following pattern of texture zeros for the Yukawa couplings:

$Y_{d}=\left[\begin{array}{lll}0 & 0 & 0 \\ 0 & 0 & 0 \\ 0 & 0 & \times\end{array}\right], \quad Y_{u}=\left[\begin{array}{lll}0 & 0 & 0 \\ 0 & 0 & 0 \\ 0 & 0 & \times\end{array}\right]$

which lead to $V_{\mathrm{CKM}}$ equal to the identity with only one nonzero quark mass in each charge sector.

\section{Vector-like quarks and generation of realistic quark masses and mixing}

A possible mechanism to generate masses for the light standard-like quarks is to introduce vector-like quarks. In our examples we specialise to the case of three down $\left(D_{L i}^{0}\right.$, $\left.D_{R i}^{0},\right)$ and three up $\left(U_{L i}^{0}, U_{R i}^{0}\right)$ vector-like isosinglet quarks. With the introduction of these additional isosinglet quarks the Yukawa interactions can now be denoted

$$
\mathcal{L}_{\mathrm{Y}}=\left[-\bar{Q}_{L i}^{0} \Phi\left(Y_{d}\right)_{i \alpha} d_{R \alpha}^{0}-\bar{Q}_{L i}^{0} \tilde{\Phi}\left(Y_{u}\right)_{i \beta} u_{R \beta}^{0}\right]+\text { h.c.; }
$$

here the index $i$ runs from 1 to 3 , as in the SM, while the indices $\alpha$ and $\beta$ cover all right-handed quark singlets of the down and up sector, respectively. The following generic bare mass terms must also be introduced in the Lagrangian:

$\mathcal{L}_{b . m .}=\left[-\bar{D}_{L j}^{0}\left(\eta_{d}\right)_{j \alpha} d_{R \alpha}^{0}-\bar{U}_{L k}^{0}\left(\eta_{u}\right)_{k \beta} u_{R \beta}^{0}\right]+$ h.c. $;$

here the indices $j$ and $k$ run over all left-handed vector-like quarks in each sector. As mentioned before, in all examples that follow $i, j$ and $k$ run from 1 to 3 and therefore $\alpha$ and $\beta$ run from 1 to 6 (obviously $D_{R i}^{0} \equiv d_{R i+3}^{0}$ and $U_{R i}^{0} \equiv$ $\left.u_{R i+3}^{0}\right)$. In what follows we extend the $Z_{6}$ discrete flavour symmetry introduced in Sect. 2.2 and we introduce a complex scalar singlet $S$. In the scalar sector the $Z_{6}$ symmetry is also imposed, but we allow for soft breaking terms like e.g., $\left(S^{2}+\right.$ $S^{* 2}$ ). These terms are crucial in order to avoid the domainwall problem. Since the symmetry is discrete, they also avoid the massless Goldstone boson. This scalar singlet will couple to the quark singlets in the following way:

$$
\begin{aligned}
\mathcal{L}_{\mathrm{g}}= & {\left[-\bar{D}_{L j}^{0}\left[\left(g_{d}\right)_{j \alpha} S+\left(g_{d}^{\prime}\right)_{j \alpha} S^{*}\right] d_{R \alpha}^{0}\right.} \\
& \left.-\bar{U}_{L k}^{0}\left[\left(g_{u}\right)_{k \beta} S+\left(g_{u}^{\prime}\right)_{k \beta} S^{*}\right] u_{R \beta}^{0}\right]+ \text { h.c. }
\end{aligned}
$$

We assume that the modulus of the vacuum expectation value of the field $S$ is of an order of magnitude higher than the electroweak scale. After spontaneous symmetry breaking the following mass terms are generated: 


$$
\begin{aligned}
\mathcal{L}_{\mathrm{M}}= & {\left[-\frac{v}{\sqrt{2}} \bar{d}_{L i}^{0}\left(Y_{d}\right)_{i \alpha} d_{R \alpha}^{0}-\frac{v}{\sqrt{2}} \bar{u}_{L i}^{0}\left(Y_{u}\right)_{i \alpha} u_{R \alpha}^{0}\right.} \\
& \left.-\bar{D}_{L i}^{0}\left(\mu_{d}\right)_{i \alpha} d_{R \alpha}^{0}-\bar{U}_{L i}^{0}\left(\mu_{u}\right)_{i \alpha} u_{R \alpha}^{0}\right]+ \text { h.c. }
\end{aligned}
$$

These terms can be written in a more compact form, as

$$
\mathcal{L}_{\mathrm{M}}=-\left(\bar{d}_{L}^{0} \bar{D}_{L}^{0}\right) \mathcal{M}_{d}\left(\begin{array}{c}
d_{R}^{0} \\
D_{R}^{0}
\end{array}\right)-\left(\bar{u}_{L}^{0} \bar{U}_{L}^{0}\right) \mathcal{M}_{u}\left(\begin{array}{c}
u_{R}^{0} \\
U_{R}^{0}
\end{array}\right)
$$

with $6 \times 6$ mass matrices, $\mathcal{M}_{d}$ and $\mathcal{M}_{u}$, denoted

$$
\mathcal{M}_{d}=\left(\begin{array}{ll}
m_{d} & \omega_{d} \\
X_{d} & M_{d}
\end{array}\right) \quad \mathcal{M}_{u}=\left(\begin{array}{cc}
m_{u} & \omega_{u} \\
X_{u} & M_{u}
\end{array}\right)
$$

\subsection{Structure of charged and neutral currents}

The matrices $\mathcal{M}_{d}$ and $\mathcal{M}_{u}$ will be diagonalised through the following unitary transformations:

$$
\begin{aligned}
& \left(\begin{array}{c}
d_{L}^{0} \\
D_{L}^{0}
\end{array}\right)=\left(\begin{array}{c}
A_{d L} \\
B_{d L}
\end{array}\right)\left(d_{L}\right) \equiv \mathcal{U}_{L}^{d} d_{L} \quad\left(\begin{array}{c}
u_{L}^{0} \\
U_{L}^{0}
\end{array}\right)=\left(\begin{array}{c}
A_{u L} \\
B_{u L}
\end{array}\right)\left(u_{L}\right) \equiv \mathcal{U}_{L}^{u} u_{L} \\
& \left(\begin{array}{c}
d_{R}^{0} \\
D_{R}^{0}
\end{array}\right) \equiv \mathcal{U}_{R}^{d} d_{R} \quad\left(\begin{array}{c}
u_{R}^{0} \\
U_{R}^{0}
\end{array}\right) \equiv \mathcal{U}_{R}^{u} u_{R}
\end{aligned}
$$

where $A_{d L}, B_{d L}, A_{u L}$ and $B_{u L}$ are $3 \times 6$ matrices; the matrices $\mathcal{U}_{L}^{d}, \mathcal{U}_{R}^{d}, \mathcal{U}_{L}^{u}$ and $\mathcal{U}_{R}^{u}$ are unitary $6 \times 6$ matrices and $\left(d_{L}\right)$, $\left(d_{R}\right),\left(u_{L}\right)$ and $\left(u_{R}\right)$ stand for the components of the six down and six up mass eigenstate quarks. Unitarity implies that

$$
\begin{aligned}
& \left(\begin{array}{c}
A_{d L} \\
B_{d L}
\end{array}\right)\left(\begin{array}{ll}
A_{d L}^{\dagger} & B_{d L}^{\dagger}
\end{array}\right)=\left(\begin{array}{ll}
A_{d L} A_{d L}^{\dagger} & A_{d L} B_{d L}^{\dagger} \\
B_{d L} A_{d L}^{\dagger} & B_{d L} B_{d L}^{\dagger}
\end{array}\right)=\left(\begin{array}{cc}
\mathbb{1}_{3 \times 3} & 0 \\
0 & \mathbb{1}_{3 \times 3}
\end{array}\right) \\
& \left(A_{d L}^{\dagger} B_{d L}^{\dagger}\right)\left(\begin{array}{c}
A_{d L} \\
B_{d L}
\end{array}\right)=A_{d L}^{\dagger} A_{d L}+B_{d L}^{\dagger} B_{d L}=\mathbb{I}_{6 \times 6}
\end{aligned}
$$

with similar relations for the up-type matrices. The charged currents are given by

$$
\begin{aligned}
\mathcal{L}_{\mathrm{W}} & =-\frac{g}{\sqrt{2}}\left(\bar{u}_{L}^{0} \gamma^{\mu} d_{L}^{0}\right) \mathbf{W}_{\mu}^{+}+\text {h.c. } \\
& =-\frac{g}{\sqrt{2}}\left(\bar{u}_{L} V \gamma^{\mu} d_{L}\right) \mathbf{W}_{\mu}^{+}+\text {h.c. }
\end{aligned}
$$

with $V=A_{u L}^{\dagger} A_{d L}$. The couplings of the $Z$ boson are of the form

$$
\begin{aligned}
\mathcal{L}_{\mathrm{Z}}= & \frac{g}{\cos \theta_{W}} Z_{\mu}\left[\frac{1}{2}\left(\bar{u}_{L} W^{u} \gamma^{\mu} u_{L}-\bar{d}_{L} W^{d} \gamma^{\mu} u_{L}\right)\right. \\
& \left.-\sin ^{2} \theta_{W}\left(\frac{2}{3} \bar{u} \gamma^{\mu} u-\frac{1}{3} \bar{d} \gamma^{\mu} d\right)\right]
\end{aligned}
$$

with $W^{d}=V^{\dagger} V$ and $W^{u}=V V^{\dagger}$. After spontaneous symmetry breaking, Eqs. (5), (6) and (7) give rise to the physical quark masses, which together with the couplings to the SMlike Higgs can be denoted

$$
\begin{aligned}
\mathcal{L}_{\mathrm{Mh}}= & -\bar{d}_{L} \mathcal{D}_{d} d_{R}-\bar{u}_{L} \mathcal{D}_{u} u_{R} \\
& -\frac{\sqrt{2} G^{+}}{v}\left[\bar{u}_{L} V \mathcal{D}_{d} d_{R}-\bar{u}_{R} \mathcal{D}_{u} V d_{L}\right] \\
& -i \frac{G^{0}}{v}\left[\bar{d}_{L} W^{d} \mathcal{D}_{d} d_{R}-\bar{u}_{L} W^{u} \mathcal{D}_{u} u_{R}\right] \\
& -\frac{h}{v}\left[\bar{d}_{L} W^{d} \mathcal{D}_{d} d_{R}+\bar{u}_{L} W^{u} \mathcal{D}_{u} u_{R}\right]+\text { h.c. }
\end{aligned}
$$

where $h$ is the Higgs field, $v$ is the vacuum expectation value of the neutral component of the Higgs doublet and $G^{+}$and $G^{0}$ are the would-be Goldstone bosons, $\mathcal{D}_{d}$ and $\mathcal{D}_{u}$ are the six by six diagonal quark mass matrices.

\subsection{Extension of the symmetry to the full Lagrangian}

In the fermion sector, as mentioned above, we introduce three down-type and three up-type vector-like quarks. In the scalar sector, in addition to the standard Higgs, we have introduced a complex scalar $S$. We extend the symmetry to the full Lagrangian, with the new fields transforming in the following way under the family symmetry:

$D_{L 1}^{0} \rightarrow e^{-3 i \tau} D_{L 1}^{0} \quad D_{L 2}^{0} \rightarrow e^{-2 i \tau} D_{L 2}^{0} \quad D_{L 3}^{0} \rightarrow e^{-i \tau} D_{L 3}^{0}$

$D_{R 1}^{0} \rightarrow e^{-2 i \tau} D_{R 1}^{0} \quad D_{R 2}^{0} \rightarrow e^{-3 i \tau} D_{R 2}^{0} \quad D_{R 3}^{0} \rightarrow D_{R 3}^{0}$

$U_{L 1}^{0} \rightarrow e^{-i \tau} U_{L 1}^{0} \quad U_{L 2}^{0} \rightarrow U_{L 2}^{0} \quad U_{L 3}^{0} \rightarrow e^{i \tau} U_{L 3}^{0}$

$U_{R 1}^{0} \rightarrow U_{R 1}^{0} \quad U_{R 2}^{0} \rightarrow e^{-i \tau} U_{R 2}^{0} \quad U_{R 3}^{0} \rightarrow e^{2 i \tau} U_{R 3}^{0} ;$

$S \rightarrow e^{i \tau} S ; \quad \tau=\frac{2 \pi}{6}$

together with the transformations for the standard-like quarks specified in Eq. (2). The singlet scalar $S$ is introduced in order to be able to obtain realistic quark masses and mixing, without breaking the symmetry at the Lagrangian level, except for the soft breaking in the scalar potential. An alternative option would be to softly break the symmetry also in the fermion sector, through the introduction of bare mass terms for the heavy fermions. Since the symmetry would be only softly broken, the model would maintain its renormalisability. We find it more appealing to have the symmetry exact in the fermion sector. We assume that the scale of spontaneous symmetry breaking of the $S$ fields is higher than the electroweak scale.

Tables 1 and 2 summarise the information on the combination of the different fermionic charges and allow one to infer what is the pattern of the mass matrices. The first three rows come from Yukawa terms of the form given by Eq. (5) and therefore are only allowed when the fermionic charge cancels the one coming from the scalar doublet. In these cases we write this charge explicitly. In the forbidden terms we 
Table 1 Down sector, summary of transformation properties. In the forbidden terms we put a bullet sign. We denote by 1 the entries corresponding to allowed bare mass terms. The fermionic charges are given for those terms that are allowed through couplings to scalar fields: $\Phi, S$ or $S^{*}$, to which we assign appropriate charges
Table 2 Up sector, summary of transformation properties. In the forbidden terms we put a bullet sign. We denote by 1 the entries corresponding to allowed bare mass terms. The fermionic charges are given for those terms that are allowed through couplings to scalar fields: $\Phi, S$ or $S^{*}$, to which we assign appropriate charges

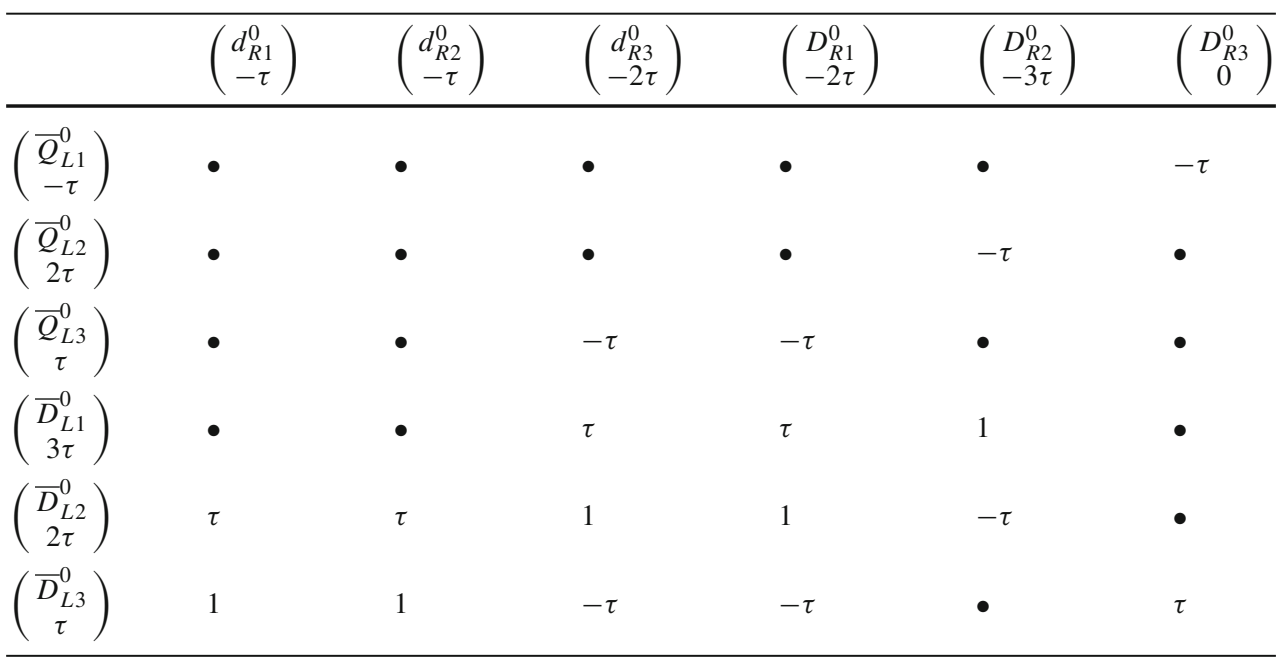

\begin{tabular}{|c|c|c|c|c|c|c|}
\hline & $\left(\begin{array}{c}u_{R 1}^{0} \\
\tau\end{array}\right)$ & $\left(\begin{array}{c}u_{R 2}^{0} \\
\tau\end{array}\right)$ & $\left(\begin{array}{c}u_{R 3}^{0} \\
0\end{array}\right)$ & $\left(\begin{array}{c}U_{R 1}^{0} \\
0\end{array}\right)$ & $\left(\begin{array}{c}U_{R 2}^{0} \\
-\tau\end{array}\right)$ & $\left(\begin{array}{c}U_{R 3}^{0} \\
2 \tau\end{array}\right)$ \\
\hline$\left(\begin{array}{c}\bar{Q}_{L 1}^{0} \\
-\tau\end{array}\right)$ & • & • & $\bullet$ & • & $\bullet$ & $\tau$ \\
\hline $\left.\begin{array}{c}\bar{Q}_{L 2}^{0} \\
2 \tau\end{array}\right)$ & • & • & $\bullet$ & • & $\tau$ & • \\
\hline$\left(\begin{array}{c}\bar{Q}_{L 3}^{0} \\
\tau\end{array}\right)$ & • & • & $\tau$ & $\tau$ & $\bullet$ & • \\
\hline$\left(\begin{array}{c}\bar{U}_{L 1}^{0} \\
\tau\end{array}\right)$ & • & • & $\tau$ & $\tau$ & 1 & • \\
\hline$\left(\begin{array}{c}\bar{U}_{L 2}^{0} \\
0\end{array}\right)$ & $\tau$ & $\tau$ & 1 & 1 & $-\tau$ & • \\
\hline$\left(\begin{array}{c}\bar{U}_{L 3}^{0} \\
-\tau\end{array}\right)$ & 1 & 1 & $-\tau$ & $-\tau$ & $\bullet$ & $\tau$ \\
\hline
\end{tabular}

put a bullet sign. The last three rows come from bare mass terms of the form given by Eq. (6) or else from couplings to the field $S$. We denote with 1 the entries corresponding to allowed bare mass terms and by the fermionic charges those terms that allow coupling to either $S$ or $S^{*}$. Again we use bullets for the forbidden terms. The introduction of these singlet scalar field provides a rationale for the choice of terms that would otherwise softly break the symmetry and would look arbitrary.

\subsection{Effective Hermitian squared mass matrix}

The $6 \times 6$ mass matrices $\mathcal{M}_{d}, \mathcal{M}_{u}$ are diagonalised through the bi-unitary transformations:

$\mathcal{U}_{L}^{d \dagger} \mathcal{M}_{d} \mathcal{U}_{R}^{d}=\mathcal{D}_{d} \equiv \operatorname{diag}\left(d_{d}, D_{d}\right)$

where $d_{d} \equiv \operatorname{diag}\left(m_{d}, m_{s}, m_{b}\right), D_{d} \equiv \operatorname{diag}\left(M_{D 1}, M_{D 2}\right.$, $\left.M_{D 3}\right)$ and with $M_{D i}$ standing for the heavy $Q=-1 / 3$ quark masses. An analogous equation holds for $\mathcal{M}_{u}$. In our examples the diagonalisation of $\mathcal{M}_{d}$ and $\mathcal{M}_{u}$ is done through an exact numerical calculation. However, in order to have an idea of the main physical features involved, it is useful to perform an approximate evaluation of $\mathcal{U}_{L}^{d}, \mathcal{U}_{L}^{u}$ and of the quark mass eigenvalues. For this purpose, it is useful to write $\mathcal{U}_{L}^{d}, \mathcal{U}_{L}^{u}$ in block form:

$\mathcal{U}_{L}=\left(\begin{array}{cc}K & R \\ S & T\end{array}\right)$

where $K, R, S, T$ are $3 \times 3$ matrices. For simplicity, we drop the indices $d$, and $u$. In Appendix A we show that the deviations of the unitarity of the matrix $K$ are naturally small:

$K K^{\dagger}=\mathbb{1}-R R^{\dagger}$

with

$R \approx \frac{\left(m X^{\dagger}+\omega M^{\dagger}\right) T}{D^{2}} \approx(m / M)$ 
and

$K^{\dagger} K=\mathbb{1}-S^{\dagger} S$

with

$S \approx\left(\frac{X m^{\dagger}+M \omega^{\dagger}}{X X^{\dagger}+M M^{\dagger}}\right) K$

The matrices $K_{d}, K_{u}$ can be evaluated from an effective Hermitian squared matrix $\mathcal{H}_{\text {eff }}$ through:

$K^{-1} \mathcal{H}_{\text {eff }} K=d^{2}$

with

$$
\begin{aligned}
\mathcal{H}_{\mathrm{eff}}= & \left(m m^{\dagger}+\omega \omega^{\dagger}\right)-\left(m X^{\dagger}+\omega M^{\dagger}\right)\left(X X^{\dagger}+M M^{\dagger}\right)^{-1} \\
& \times\left(X m^{\dagger}+M \omega^{\dagger}\right) .
\end{aligned}
$$

The derivation of $\mathcal{H}_{\text {eff }}$ is given in Appendix A.

\subsection{Qualitative analysis of quark mixing}

We analyse here the qualitative features of quark mixing among standard quarks. As we have seen in the previous section, in this framework the $3 \times 3 V_{\mathrm{CKM}}$ matrix is generated through the mixing of standard quarks with vectorlike quarks, leading to an effective Hermitian squared mass matrix given by Eq. (24). For simplicity, we will assume that the dominant contribution to $V_{\mathrm{CKM}}$ arises from the diagonalisation of the down quark sector.

We assume that the $6 \times 6$ down quark mass matrix has a Froggatt-Nielsen structure [50], with its elements parametrised in terms of a small parameter $\lambda$, with all other parameters of order one:

$\mathcal{M}_{d}=\mu\left(\begin{array}{cccccc}0 & 0 & 0 & 0 & 0 & \lambda^{2} z \\ 0 & 0 & 0 & 0 & \lambda y & 0 \\ 0 & 0 & 1 & \lambda x & 0 & 0 \\ 0 & 0 & \frac{C_{1}}{\lambda} & \frac{D_{1}}{\lambda^{2}} & 0 & 0 \\ \frac{A_{2}}{\lambda} & \frac{B_{2}}{\lambda} & \frac{C_{2}}{\lambda} & 0 & \frac{D_{2}}{\lambda^{2}} & 0 \\ \frac{A_{3}}{\lambda} & 0 & \frac{C_{3}}{\lambda} & 0 & 0 & \frac{D_{3}}{\lambda^{2}}\end{array}\right)$,

where $\mu \approx m_{b}$. Using Eq. (24) one can derive that $\mathcal{H}_{\text {eff }}$ has the following structure:

$\mathcal{H}_{\mathrm{eff}} \sim\left(\begin{array}{ccc}\lambda^{6} r z^{2} & \lambda^{5} u y z & -\lambda^{3} c_{3} z \\ \lambda^{5} u^{*} y z & \lambda^{4} r^{\prime} y^{2} & -\lambda^{2} c_{2} y \\ -\lambda^{3} c_{3}^{*} z & -\lambda^{2} c_{2}^{*} y & 1-\lambda^{2} \hat{r}\end{array}\right)$

with

$$
\begin{aligned}
r & =\left|a_{3}\right|^{2}+\left|c_{3}\right|^{2} \\
r^{\prime} & =\left|a_{2}\right|^{2}+\left|b_{2}\right|^{2}+\left|c_{2}\right|^{2}
\end{aligned}
$$

$$
\begin{aligned}
\hat{r} & =\left|c_{1}\right|^{2}+\left|c_{2}\right|^{2}+\left|c_{3}\right|^{2}+\left(c_{1}+c_{1}^{*}\right) x \\
u & =a_{2}^{*} a_{3}+c_{2}^{*} c_{3}, \\
a_{i} & =\frac{A_{i}}{D_{i}}, \quad b_{i}=\frac{B_{i}}{D_{i}}, \quad c_{i}=\frac{C_{i}}{D_{i}} .
\end{aligned}
$$

Using, Eq. (24) and taking into account that $\mathcal{H}_{\text {eff }}=$ $V_{\mathrm{CKM}} d_{d}^{2} V_{\mathrm{CKM}}^{\dagger}$ with $d_{d}^{2}=\operatorname{diag}\left(m_{d}^{2}, m_{s}^{2}, m_{b}^{2}\right)$, one obtains

$\left|V_{c b}\right| \approx K_{23} \lambda^{2} \quad\left|V_{u b}\right| \approx K_{13} \lambda^{3}$

where $K_{i j}$ are order one, namely $K_{23} \equiv\left(c_{2} y\right), K_{13} \equiv\left(c_{3} z\right)$. Identifying $\lambda$ with the Cabibbo parameter, one sees that the experimental values of $\left|V_{u b}\right|,\left|V_{c b}\right|$ can be obtained. A more detailed analysis can show that the full $V_{\mathrm{CKM}}$ matrix can be obtained, in agreement with experiment. In this section, we illustrate in a qualitative way how one can obtain a correct $V_{\mathrm{CKM}}$ at low energies starting from a $6 \times 6$ quark mass matrix parametrised by powers of a small parameter $\lambda$ and order one parameters. In the next section, we give a realistic example with an exact diagonalisation of the $6 \times 6$ quark mass matrices.

\section{Realistic examples}

Following the notation in Eq. (10) we present one full realistic example; mass matrices are given at the $M_{Z}$ scale in units of GeV. Among the matrices coming from electroweak symmetry breaking we have: (i) the mass matrices that connect light (ordinary) quarks among themselves $m_{d}$ and $m_{u}$; they should contain the dominant contributions to the $b$ and $t$ quarks masses, allowed by the symmetry in Eq. (2),

$m_{d}=\left(\begin{array}{llc}0 & 0 & 0 \\ 0 & 0 & 0 \\ 0 & 0 & 3.07015\end{array}\right), \quad m_{u}=\left(\begin{array}{ccc}0 & 0 & 0 \\ 0 & 0 & 0 \\ 0 & 0 & 185.142\end{array}\right)$,

and (ii) the upper right off-diagonal blocks of the quark mass matrices that read

$\omega_{d}=\left(\begin{array}{ccc}0 & 0 & 0.061403 \\ 0 & 0.39912 & 0 \\ 0.39912 & 0 & 0\end{array}\right)$,

$\omega_{u}=\left(\begin{array}{ccc}0 & 0 & 0.00925708 \\ 0 & 3.70283 & 0 \\ 185.142 & 0 & 0\end{array}\right)$.

The matrices in Eqs. (29) and (30) are proportional to the Higgs vacuum expectation value; therefore its matrix elements should be of the same order of magnitude as the corresponding bottom or top mass, or smaller. The heavy vectorlike quarks mass sectors $M_{d}$ and $M_{u}$ are 


$$
\begin{aligned}
M_{d} & =\left(\begin{array}{ccc}
767.538 & 0 & 0 \\
0 & 1535.08 & 0 \\
0 & 0 & 1842.09
\end{array}\right), \\
M_{u} & =\left(\begin{array}{ccc}
1295.99 & 0 & 0 \\
0 & 1481.13 & 0 \\
0 & 0 & 2221.7
\end{array}\right) .
\end{aligned}
$$

It is clear that the light masses agree with the light masses of the SM [51,52]. From the diagonalisation of $\mathcal{M}_{d}$ and $\mathcal{M}_{u}$ we also obtain the non-unitary $6 \times 6 \mathrm{CKM}$ matrix $V$. Its moduli are given by

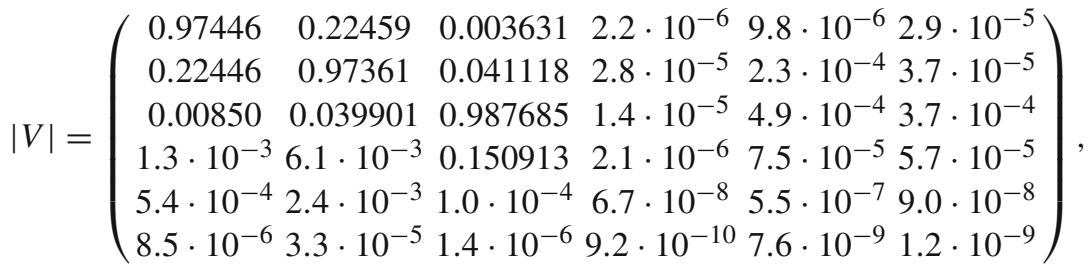

These matrices fix approximately the masses of the new heavy vector-like singlet quarks. Finally, the other matrices that connect the heavy sector with the light one are and the arguments of its matrix elements are given in leading order by

$\arg (V)=\left(\begin{array}{cccccc}0 & 6.4 \cdot 10^{-4} & -1.197 & -8.2 \cdot 10^{-3} & -3.109 & -2.3 \cdot 10^{-3} \\ \pi & 0 & 0 & 1.22 & 0.668 & 0.666 \\ -0.393 & \pi+0.0188 & 0 & -1.93 & -2.54 & -2.13 \\ 1.63 & -1.10 & 2.02 & 0.091 & -0.516 & -0.107 \\ 1.57 & -1.56 & -1.56 & -0.345 & -0.895 & -0.896 \\ -1.09 & 1.55 & 1.57 & 2.79 & 2.25 & 2.17\end{array}\right)$.

$$
\begin{aligned}
& X_{d}=\left(\begin{array}{ccc}
0 & 0 & -115.131 \\
-262.498 i & 46.0523 & 460.523-230.261 i \\
486.312 & 0 & 368.418
\end{array}\right) \text {, } \\
& X_{u}=\left(\begin{array}{ccc}
0 & 0 & 68.4281 \\
-212.913 & -185.142 & 0 \\
416.569 & 0 & 0
\end{array}\right) \text {. }
\end{aligned}
$$

The scale of the matrices in Eqs. (31), (32) and (33) is, for most entries similar or higher than the electroweak scale: the off-diagonal matrices being always smaller than the heavy mass matrix sectors in order to be the responsible of giving mass to the light quark masses and generating the CKM mixing. Following the standard diagonalisation of $\mathcal{M}_{d}$ and $\mathcal{M}_{u}$ the quark mass spectrum (in $\mathrm{GeV}$ at the $M_{Z}$ scale) is

$$
\left(\begin{array}{c}
m_{d} \\
m_{s} \\
m_{b} \\
m_{D_{1}} \\
m_{D_{2}} \\
m_{D_{3}}
\end{array}\right)=\left(\begin{array}{c}
0.0027 \\
0.068 \\
2.9 \\
775 \\
1621 \\
1957
\end{array}\right), \quad\left(\begin{array}{c}
m_{u} \\
m_{c} \\
m_{t} \\
m_{U_{1}} \\
m_{U_{2}} \\
m_{U_{3}}
\end{array}\right)=\left(\begin{array}{c}
0.0011 \\
0.69 \\
173 \\
1313 \\
1507 \\
2261
\end{array}\right)
$$

This generalised non-unitary CKM matrix $V$ deserves several comments:

1. The first upper left $3 \times 3$ block reproduces to a great extent the SM CKM mixing matrix, including the phases.

2. It is remarkable that all the moduli-except $\left|V_{t b}\right|-$ of the $3 \times 3$ light sector of $V$ agree with the SM fitted values within $1.5 \sigma$, in fact most are within $1 \sigma$.

3. A very important difference is in the element $\left|V_{t b}\right|$, which is incompatible with the SM value. Disentangling this value from the SM one is certainly an experimental challenge for single top production.

4. Looking at the four independent phases that can be defined in the $3 \times 3$ light sector $\beta, \gamma, \beta_{s}$ and $\chi^{\prime}$-related to the phases of $V_{t d}, V_{u b}, V_{t s}$ and $V_{u s}$, see Refs. [35,53]we cannot find any relevant difference among the phases of this example and the phases of the SM. We get in our model

$\beta=0.393, \gamma=1.197, \beta_{s}=0.0188, \chi^{\prime}=0.000636$.

5. Since the new quark singlets do not couple directly to the $\mathrm{SU}(2)_{L}$ gauge bosons, in the limit of no mixing among 
chiral and vector-like singlet quarks only the upper left $3 \times 3$ sector of $V$ is different from zero. This explains the smallness of the entries in the other sectors of $V$ mass $m_{U_{1}}=1313 \mathrm{GeV}: U_{1} \rightarrow t Z$ and $U_{1} \rightarrow t h$. The moduli of the matrix elements that control the FCNC in the down sector are given by $W^{d}=V^{\dagger} V$ :

$$
\left|W^{d}\right|=\left(\begin{array}{cccccc}
1 & 1.4 \cdot 10^{-8} & 3.0 \cdot 10^{-8} & 6.0 \cdot 10^{-16} & 6.3 \cdot 10^{-5} & 2.3 \cdot 10^{-5} \\
1.4 \cdot 10^{-8} & 1 & 1.4 \cdot 10^{-7} & 2.8 \cdot 10^{-5} & 2.4 \cdot 10^{-4} & 5.6 \cdot 10^{-5} \\
3.0 \cdot 10^{-8} & 1.4 \cdot 10^{-7} & 1 & 1.3 \cdot 10^{-5} & 4.9 \cdot 10^{-4} & 3.7 \cdot 10^{-4} \\
6.0 \cdot 10^{-6} & 2.8 \cdot 10^{-5} & 1.3 \cdot 10^{-5} & 9.6 \cdot 10^{-10} & 1.3 \cdot 10^{-8} & 6.3 \cdot 10^{-9} \\
6.3 \cdot 10^{-5} & 2.4 \cdot 10^{-4} & 4.9 \cdot 10^{-4} & 1.3 \cdot 10^{-8} & 3.0 \cdot 10^{-7} & 1.9 \cdot 10^{-7} \\
2.3 \cdot 10^{-5} & 5.6 \cdot 10^{-5} & 3.7 \cdot 10^{-4} & 6.3 \cdot 10^{-9} & 1.9 \cdot 10^{-7} & 1.4 \cdot 10^{-7}
\end{array}\right)
$$

and indicates that the elements $V_{U_{i} d}, V_{U_{i} s}$ and $V_{U_{i} b}$ can induce new physics effects that could appear for example in $b \rightarrow d, b \rightarrow s$ or $s \rightarrow d$ transitions. Transitions induced by $V_{u D_{i}}, V_{c D_{i}}$ and $V_{t D_{i}}$ should be smaller than the latter.

In summary, the CKM sector of our mixing matrix $V$ reproduces very well the SM case except for a minor but definite deviation in $V_{t b}$ at the $1 \%$ level.

\subsection{The FCNC structure}

In spite of the great similarity between the $3 \times 3$ sector of our mixing matrix $V$ and the SM CKM matrix, we know that in this model we will have FCNC at tree level both in the up and in the down sectors. Of course we expect to have these FCNC highly suppressed, but to check these expectations we present the matrices that control these FCNC both in the couplings to the $Z$ and to the Higgs $h$ bosons. The moduli of the matrix elements that control the FCNC in the up sector are given by $W^{u}=V V^{\dagger}$ :

$$
\left|W^{u}\right|=\left(\begin{array}{llll}
1 & 1.1 \cdot 10^{-8} & 2.9 \cdot 10^{-17} & 4.5 \cdot 10^{-18} \\
1.1 \cdot 10^{-8} & 1 & 2.2 \cdot 10^{-17} & 5.7 \cdot 10^{-17} \\
2.9 \cdot 10^{-17} & 2.2 \cdot 10^{-17} & 0.977 & 0.149 \\
4.5 \cdot 10^{-18} & 5.7 \cdot 10^{-17} & 0.149 & 2.28 \cdot 10^{-2} \\
4.7 \cdot 10^{-6} & 2.4 \cdot 10^{-3} & 1.7 \cdot 10^{-16} & 2.6 \cdot 10^{-17} \\
4.0 \cdot 10^{-6} & 3.4 \cdot 10^{-5} & 2.2 \cdot 10^{-18} & 3.3 \cdot 10^{-19}
\end{array}\right.
$$

In the light sector $\left|W_{u c}^{u}\right|=1.1 \cdot 10^{-8}$ is too small to be in conflict with $D^{0}-\bar{D}^{0}$ mixing or $D^{0} \rightarrow \mu \bar{\mu}$. Values like $\left|W_{q t}^{u}\right| \sim 10^{-17}$ make $t \rightarrow Z u, Z c$ extremely suppressed. The reminiscent of $V_{t b} \neq 1$ is here $\left|W_{t t}^{u}\right|=0.977$ another challenging deviation from the SM to be checked more likely in loops. It is important to realise that the light $3 \times 3 \mathrm{sec}-$ tor resembles very much the identity matrix $\mathbb{I}_{3 \times 3}$ except for $\left|W_{t t}^{u}\right|$. Values like $\left|W_{U_{1} t}^{u}\right|=0.149$ will dictate the neutral current dominant decay channels of the heavy quark $U_{1}$ of
Owing to the fact that $W^{d}$ can induce $K^{0}-\bar{K}^{0}, B_{d}^{0}-\bar{B}_{d}^{0}$ and $B_{s}^{0}-\bar{B}_{s}^{0}$ mixing at tree level through $Z$ exchange, the offdiagonal elements $W_{d s}^{d}, W_{d b}^{d}$ and $W_{s b}^{d}$ cannot be too large. But as we can see they are well below (two to three orders of magnitude) the values from previous analyses $[32,33]$ that avoid conflicting with meson mixing constraints. The bottom line is that the $3 \times 3$ light sector of $\left|W^{d}\right|$ is very well approximated by $\mathbb{1}_{3 \times 3}$.

But this is not the end of the story. We have now an enlarged $6 \times 6 \mathrm{CKM}$ matrix $V$ with elements connecting light and heavy quarks of order $10^{-3}$ to $10^{-4}$. These matrix elements enter into the loops that generate FCNC with heavy quarks running inside the loop and with several Inami-Lim (IL) functions [54] growing with the square of the heavy quark masses. So, a priori, one has to check that the product of these heavy masses with these suppressed couplings does not spoil the great success of the SM in FCNC processes. At this point it is worthwhile recalling that we have fixed our model with vector-like quarks by demanding that a particular texture mass structure, imposed by symmetries, should reproduce the light quark masses and the dominant

$$
\left.\begin{array}{ll}
4.7 \cdot 10^{-6} & 4.0 \cdot 10^{-6} \\
2.4 \cdot 10^{-3} & 3.4 \cdot 10^{-5} \\
1.7 \cdot 10^{-16} & 2.2 \cdot 10^{-18} \\
2.6 \cdot 10^{-17} & 3.3 \cdot 10^{-19} \\
5.8 \cdot 10^{-6} & 8.1 \cdot 10^{-8} \\
8.1 \cdot 10^{-8} & 1.1 \cdot 10^{-9}
\end{array}\right)
$$

CKM mixing matrix. On the other hand, the symmetry does not impose any constraint on the product of heavy masses square and mixing between light and heavy fermions. Therefore we should check the relevant constraints.

\subsection{Loop FCNC constraints}

In general the structure of FCNC at one loop level in this model can be quite involved. But, as we have seen, the tree level flavour changing coupling are very much suppressed. So 
let us comment on the different processes where, as we will see, tree level FCNC contributions can be safety neglected:

1. The contributions that go with $W_{i j}^{u}$ and $W_{i j}^{d}$ in FCNC tree level contributions to $\Delta F=1$ processes like $B_{q} \rightarrow \mu \bar{\mu}$. These NP pieces are proportional to $W_{b q}^{d}$ to be compared with $\alpha_{e m} V_{t b}^{*} V_{t q}$ times an IL function of order one coming from the SM piece. The transitions where these NP contributions are the largest ones are rare kaon decays where we have $W_{d s}^{d} / \alpha_{e m} V_{t d}^{*} V_{t s} \lesssim 10^{-2}$. So for example $K^{+} \rightarrow \pi^{+} \nu \bar{v}$ or $K_{L} \rightarrow \mu \bar{\mu}$ are not affected by these tree level contributions. The corresponding decays in the $B$ meson systems are even less affected.

2. The contributions that go with $\left(W_{i j}^{u}\right)^{2}$ and $\left(W_{i j}^{d}\right)^{2}$ in FCNC tree level contributions to $\Delta F=2$ processes like $D^{0}-\bar{D}^{0}$ or $K^{0}-\bar{K}^{0}, B_{d}^{0}-\bar{B}_{d}^{0}$ and $B_{s}^{0}-\bar{B}_{s}^{0}$. In these cases, we are neglecting $W_{d s}^{d}$ with respect to $\sqrt{\alpha_{e m}} V_{t d}^{*} V_{t s}$ and similarly for the $B_{d}, B_{s}$ and $D$ neutral meson systems. The largest NP correction appears in the kaon case and is of order $10^{-6}$ times the SM contribution.

3. When the tree level FCNC are small, Barenboim and Botella [55] showed that the leading NP contribution to meson mixing, induced by this FCNC enters at order $\alpha_{e m} W_{d s}^{d} V_{t d}^{*} V_{t s}$, to be compared with $\alpha_{e m}\left(V_{t d}^{*} V_{t s}\right)^{2}$. So, in our example, these contributions are at most of order $10^{-4}$. Again, we can neglect these contributions.

Taking into account the previous considerations, we can analyse all the $\Delta F=1,2$ processes neglecting the tree level FCNC effects.

\subsection{1 $\Delta F=2$ pure loop constraints}

The neutral meson mixing is therefore dominated by the box diagrams that generalise the SM one, with all species of heavy quarks plus the top and the charm quarks - or the bottom and the strange quarks for $D^{0}-\bar{D}^{0}$ mixing-running inside the loop. ${ }^{1}$ If we define as usual $\lambda_{q q^{\prime}}^{a}=V_{a q^{\prime}}^{*} V_{a q}$-for mesons with down quarks-, the dominant corrections to the mixing $\left(M_{12}\right)_{q q^{\prime}}$ of the meson with quark content $\left(q q^{\prime}\right)$, with respect to the SM box diagram with internal top quarks, can be written as

\footnotetext{
1 Note that in the SM, the Inami-Lim function that appears in the box runs for $c$ and $t$ quarks after using unitarity $3 \times 3$. In our case we have for the kaon system, for example, $\sum_{i=1}^{6} V_{i d} V_{i s}^{*}=\left(V^{\dagger} V\right)_{d s}=W_{d s}^{d}$, so there is an additional contribution-to what we are considering in the main text-proportional to the quark $u$ contribution and to $W_{d s}^{d}$, and therefore negligible. So we can extend the sum from $c, t$ to $c, t, U_{1}, U_{2}, U_{3}$.
}

$$
\begin{aligned}
& \frac{\left(M_{12}\right)_{q q^{\prime}}}{\left(M_{12}^{S M}\right)_{q q^{\prime}}} \sim 1+\sum_{i=1}^{3}\left(\frac{\lambda_{q q^{\prime}}^{U_{i}}}{\lambda_{q q^{\prime}}^{t}}\right)^{2} \frac{S\left(x_{U_{i}}\right)}{S\left(x_{t}\right)} \\
& +2 \sum_{i=1}^{3}\left(\frac{\lambda_{q q^{\prime}}^{U_{i}}}{\lambda_{q q^{\prime}}^{t}}\right) \frac{S\left(x_{U_{i}}, x_{t}\right)}{S\left(x_{t}\right)} \\
& +2 \sum_{i<j}^{3} \frac{\left(\lambda_{q q^{\prime}}^{U_{i}}, \lambda_{q q^{\prime}}^{U_{j}}\right)}{\left(\lambda_{q q^{\prime}}^{t}\right)^{2}} \frac{S\left(x_{U_{i}}, x_{U_{j}}\right)}{S\left(x_{t}\right)}+\cdots
\end{aligned}
$$

where $S\left(x_{t}\right), S\left(x_{U_{i}}, x_{t}\right)$ are the IL functions defined as in [53] and $x_{t}, x_{U_{i}}=\left(m_{t} / M_{W}\right)^{2},\left(m_{U_{i}} / M_{W}\right)^{2}$. In our case we have for the first and second corrections in the kaon system

$\left|\frac{\lambda_{s d}^{U_{1}}}{\lambda_{s d}^{t}}\right|^{2} \frac{S\left(x_{U_{1}}\right)}{S\left(x_{t}\right)}=1.6 \times 10^{-2}$,

$2\left|\frac{\lambda_{s d}^{U_{1}}}{\lambda_{s d}^{t}}\right| \frac{S\left(x_{U_{1}}, x_{t}\right)}{S\left(x_{t}\right)}=0.13$.

Including the charm and the leading QCD corrections, and defining the corrections with respect the SM,

$\begin{aligned} \Delta\left(P^{0}\right) & =\left|\left(\frac{M_{12}}{M_{12}^{S M}}\right)_{P^{0}}\right|-1, \\ \delta \phi\left(P^{0}\right) & =\left(\frac{\arg \left(M_{12}\right)}{\arg \left(M_{12}^{S M}\right)}\right)_{P^{0}}-1,\end{aligned}$

we get for the $K^{0}, B_{d}$ and $B_{s}$ mixings

$\left(\begin{array}{l}\Delta\left(K^{0}\right) \\ \Delta\left(B_{d}^{0}\right) \\ \Delta\left(B_{s}^{0}\right)\end{array}\right)=\left(\begin{array}{l}0.02 \\ 0.12 \\ 0.12\end{array}\right),\left(\begin{array}{c}\delta \phi\left(K^{0}\right) \\ \delta \phi\left(B_{d}^{0}\right) \\ \delta \phi\left(B_{s}^{0}\right)\end{array}\right)=\left(\begin{array}{l}0.12 \\ 0.01 \\ 0.01\end{array}\right)$

We conclude that these kinds of models are compatible with the actual analysis beyond the SM, but they can have sizeable effects. For example this model does not modify $\Delta M_{B_{d}} / \Delta M_{B_{s}}$ but can give $12 \%$ corrections to $\Delta M_{B_{d}}$ and $\Delta M_{B_{s}}$. Furthermore, $\epsilon_{K}$ can have $12 \%$ corrections.

\subsection{2 $\Delta F=1$ pure loop constraints}

In these processes-for example $q \rightarrow q^{\prime} \mu \bar{\mu}$ - the corrections to the top dominated SM amplitudes (when necessary, charm has to be taken into account) are given by

$$
\begin{aligned}
& \frac{A\left(q \rightarrow q^{\prime} \mu \bar{\mu}\right)}{A\left(q \rightarrow q^{\prime} \mu \bar{\mu}\right)_{S M}} \sim 1+\sum_{i=1}^{3} \frac{\lambda_{q q^{\prime}}^{U_{i}} Y\left(x_{U_{i}}\right)}{\lambda_{q q^{\prime}}^{t} Y\left(x_{t}\right)} \\
& -\sum_{i, j=c, t, U_{1}, U_{2}, U_{3}} \frac{V_{i q^{\prime}}^{*}\left(W^{u}-I\right)_{i j} V_{j q} N\left(x_{i}, x_{j}\right)}{\lambda_{q q^{\prime}}^{t} Y\left(x_{t}\right)} .
\end{aligned}
$$


The IL functions $Y\left(x_{U_{i}}\right)$ grow with the square of the quark mass $U_{i}$-also $N\left(x_{U_{i}}, x_{U_{i}}\right)$. This fact, apparently, will make the second term more relevant than the corresponding one in $\Delta F=2$ processes: here there is only a $\lambda_{q q^{\prime}}^{U_{i}} / \lambda_{q q^{\prime}}^{t}$ suppression and not a $\left(\lambda_{q q^{\prime}}^{U_{i}} / \lambda_{q q^{\prime}}^{t}\right)^{2}$ suppression as in Eq. (40). But the $W_{U_{i} U_{i}}^{u}$ are very small-the new quarks are singlets under $\mathrm{SU}(2)_{L}-$, therefore the last term has a piece enforcing decoupling and cancelling partially the second piece. ${ }^{2}$ For more details one can see Refs. [56-59]. A similar structure appears in $q \rightarrow q^{\prime} v \bar{\nu}$. If we define, as in the $\Delta F=2$ processes, the deviation from the SM model

$r(A \rightarrow B)=\frac{\Gamma(A \rightarrow B)}{\Gamma(A \rightarrow B)_{S M}}-1$,

we get

$$
\begin{aligned}
& r\left(K_{L} \rightarrow \mu \bar{\mu}\right)_{S D}=0.32, \quad r\left(B_{d} \rightarrow \mu \bar{\mu}\right)=0.31, \\
& \quad r\left(B_{S} \rightarrow \mu \bar{\mu}\right)=0.30, \\
& r\left(K^{+} \rightarrow \pi^{+} \nu \bar{v}\right)=0.20, \quad r\left(B^{+} \rightarrow \pi^{+} \nu \bar{\nu}\right)=0.21, \\
& \quad r\left(B^{+} \rightarrow K^{+} \nu \bar{v}\right)=0.20 .
\end{aligned}
$$

Some of these predictions can be definitely excluded or verified very soon by the LHC experiments. One example is the effect predicted for $B_{s} \rightarrow \mu \bar{\mu}$, which deviates significantly from the SM value [60,61]. If the $R_{K}$ and $P_{5}^{\prime}$ anomalies [62] have a common origin and therefore are both related to lepton universality breaking, it is clear that the scheme developed here cannot explain them. In this context only with the introduction of vector-like fermions in the leptonic sector can one produce lepton universality breaking.

We also have analysed other loop mediated processes and, for example, we get for the oblique corrections $[63,64]$

$\Delta T=0.22, \Delta S=0.06, \Delta U=0.003$.

\subsection{The heavy vector-like quark decay channels}

In our model the new heavy quarks decay mainly throughout charged or neutral currents. The decays can be characterised by $Q_{j} \rightarrow q_{i} B$. where $Q_{j}=U_{j}, D_{j}$, is the new heavy fermion, $B=Z, h, W^{ \pm}$and $q_{i}$ the final state fermion, namely $u, c, t, d, s$ or $b$. The different partial decay widths can be written as

$$
\Gamma\left(Q_{j} \rightarrow q_{i} B\right)=\xi_{B} \frac{m_{Q_{j}}^{2}}{32 \pi v^{2}}\left|X_{i j}^{B, Q_{j}}\right|^{2} f_{B}\left(x_{j}^{B}, r_{i j}\right)
$$

\footnotetext{
2 Note that this term is usually overlooked in the literature.
}

where

$x_{j}^{B}=\frac{M_{B}^{2}}{m_{Q_{j}}^{2}}, r_{i j}=\frac{m_{q_{i}}^{2}}{m_{Q_{j}}^{2}}, \xi_{Z}=\xi_{h}=1, \xi_{W^{ \pm}}=2$,

and $X_{i j}^{B, Q_{j}}=\left\{\begin{array}{l}V_{i j} \text { for } B=W^{-} \\ V_{j i} \text { for } B=W^{+} \\ W_{i j}^{Q} \text { for } B=h, Z\end{array}\right.$

and finally

$f_{W^{ \pm}}(x, r)=f_{Z}(x, r), \quad f_{h}(x, r) \sim f_{Z}(x, r) \sim 1$,

where the last two relations are valid when $m_{q_{i}}, M_{Z}, M_{W}, M_{h}$ $\ll m_{Q_{j}}$. Detailed formulae are included in Appendix B. In this regime, to a very good approximation [25],

$\Gamma\left(Q_{j} \rightarrow q_{i} Z\right) \simeq \Gamma\left(Q_{j} \rightarrow q_{i} h\right)$

Furthermore, under the same conditions, we also have

$\Gamma\left(U_{j} \rightarrow d_{i}^{\prime} W\right) \simeq 2 \frac{\left|V_{j i}\right|^{2}}{\left|W_{i j}^{u}\right|^{2}} \Gamma\left(U_{j} \rightarrow u_{i} Z\right)$,
$\Gamma\left(D_{j} \rightarrow u_{i}^{\prime} W\right) \simeq 2 \frac{\left|V_{i j}\right|^{2}}{\left|W_{i j}^{d}\right|^{2}} \Gamma\left(D_{j} \rightarrow d_{i} Z\right)$,

for $q_{i}$ and $q_{i}^{\prime}$ in the same generation, as suggested by the subindex $i$ : for example $c$ and $s$. It turns out that, under reasonable conditions, as explained in Appendix B, it is quite common to have $\left|V_{j i}\right|^{2} \sim\left|W_{i j}^{u}\right|^{2}$ and $\left|V_{i j}\right|^{2} \sim\left|W_{i j}^{d}\right|^{2}$. Therefore we also have

$\Gamma\left(Q_{j} \rightarrow q_{i}^{\prime} W\right) \simeq 2 \Gamma\left(Q_{j} \rightarrow q_{i} Z\right)$

Although many searches for new heavy quarks assume $\Gamma\left(Q_{j} \rightarrow q_{i} Z\right): \Gamma\left(Q_{j} \rightarrow q_{i} h\right): \Gamma\left(Q_{j} \rightarrow q_{i}^{\prime} W\right)=$ $1: 1: 2$ and $B_{r}\left(Q_{j} \rightarrow q_{i} Z\right)+B_{r}\left(Q_{j} \rightarrow q_{i} h\right)+$ $B_{r}\left(Q_{j} \rightarrow q_{i}^{\prime} W\right) \simeq 1$, the latter is unjustified and the total $Q_{j}$ decay width can be distributed among the decay channels to different generations, while the $1: 1: 2$ pattern of branching ratios " per generation" is maintained [30]. This fact happens in the present model and we show in Tables 3 and 4 the dominant decay channels of the heavy quarks.

For example, $D_{1}$ - the lightest of the heavy vector-like quarks in this model-has around $20 \%$ of its branching ratio to decay channels in the third generation and some $80 \%$ decaying to the second generation $(Z s, h s, W c)$. These are not the most common ways of searching for these down-type vector-like quarks, although the different results by ATLAS and CMS can be adapted. Note that neither the decay to the third family is the dominant one nor there is a unique family entering in the dominant decay products. Similar patterns 
Table 3 Decays of new down-type quarks

\begin{tabular}{lllrrrrrrrr}
\hline & Width (MeV) & \multicolumn{1}{l}{ Branching ratio to channel (\%) } \\
\cline { 3 - 11 } & & $Z d$ & \multicolumn{1}{c}{$Z s$} & \multicolumn{1}{c}{$Z$ Z } & \multicolumn{1}{c}{$h d$} & \multicolumn{1}{c}{$h s$} & \multicolumn{1}{c}{$h b$} & $W u$ & $W c$ & $W t$ \\
\hline$D_{1}$ & $2.9 \times 10^{-4}$ & 0.9 & 20.3 & 4.4 & 0.9 & 19.3 & 4.2 & 0.2 & 40.7 & 8.8 \\
$D_{2}$ & 0.81 & 0.3 & 4.9 & 20.4 & 0.3 & 4.9 & 20.2 & 0 & 8.9 & 40.0 \\
$D_{3}$ & 0.69 & 0 & 0.5 & 24.9 & 0 & 0.5 & 24.7 & 0.3 & 0.5 & 48.1 \\
\hline
\end{tabular}

\begin{tabular}{lllllllllll}
\hline & Width $(\mathrm{GeV})$ & \multicolumn{8}{l}{ Branching ratio to channel (\%) } \\
\cline { 3 - 10 } & & $Z u$ & $Z c$ & $Z t$ & $h u$ & $h c$ & $h t$ & $W d$ & $W s$ & $W b$ \\
\hline$U_{1}$ & 32.9 & 0 & 0 & 23.9 & 0 & 0 & 24.7 & 0 & 0 & 51.4 \\
$U_{2}$ & $1.3 \times 10^{-2}$ & 0 & 25.1 & 0 & 0 & 24.7 & 0 & 2.5 & 47.6 & 0 \\
$U_{3}$ & $8.6 \times 10^{-6}$ & 0.3 & 24.7 & 0 & 0.3 & 24.5 & 0 & 3.2 & 46.8 & 0 \\
\hline
\end{tabular}

Table 4 Decays of new up-type quarks
Let us construct a second solution, labelled "(2)" that differs by the real numbers $\rho_{d}$ and $\rho_{u}$ :

$\mathcal{M}_{d}^{(2)}=\left(\begin{array}{cc}m_{d} & \omega_{d} \\ \rho_{d} X_{d}^{(1)} & \rho_{d} M_{d}^{(1)}\end{array}\right), \mathcal{M}_{u}^{(2)}=\left(\begin{array}{cc}m_{u} & \omega_{u} \\ \rho_{u} X_{u}^{(1)} & \rho_{u} M_{u}^{(1)}\end{array}\right)$.

Now, for $\rho_{d}, \rho_{u}>1$ we obtain another solution which reproduces "essentially" the same light quark masses and mixings, while deviations from the SM decouple as $\rho_{d}$ and $\rho_{u}$ are increased. The basic equations are Eqs. (19)-(24). Equation (24) shows that $\mathcal{H}_{\text {eff }}^{u}$ does not change at leading order in going from $\mathcal{M}_{u}^{(1)}$ to $\mathcal{M}_{u}^{(2)}$ and so $K_{u}^{(2)} \sim K_{u}^{(1)}$. At the same time Eqs. (20)-(22) tell us that $R_{u}^{(1)}$ and $S_{u}^{(1)}$ scale as $R_{u}^{(2)} \sim \rho_{u}^{-1} R_{u}^{(1)}$ and $S_{u}^{(2)} \sim \rho_{u}^{-1} S_{u}^{(1)}$, which leads to a small change in $K_{u}^{(2)}$ taking it closer to unitarity. The invariance of $\mathcal{H}_{\text {eff }}^{u(1)} \rightarrow \mathcal{H}_{\text {eff }}^{u(2)}$ under the scaling in Eq. (54) is at the origin of the fact that, once we have a solution of the type presented here, we have a continuum of solutions with essentially the same light quark masses and mixing and with heavy quarks much heavier and more decoupled. It remains to check that the effects from heavy quarks running inside the loops also decouple. This can easily be seen by realizing that our CKM matrix can also be written

$V=\left(\begin{array}{c}K_{u}^{\dagger} \\ R_{u}^{\dagger}\end{array}\right)\left(\begin{array}{ll}K_{d} & R_{d}\end{array}\right)=\left(\begin{array}{cc}K_{u}^{\dagger} K_{d} & K_{u}^{\dagger} R_{d} \\ R_{u}^{\dagger} K_{d} & R_{u}^{\dagger} R_{d}\end{array}\right)$

characteristics of the low mass sector, have the feature that New Physics effects smoothly decouple in the low energy phenomenology. In order to see how this decoupling arises, let us consider Eq. (10) and label our explicit example in Eqs. (29)-(33) with a superscript "(1)", i.e., the matrices in Eqs. (29)-(33) are

$\mathcal{M}_{d}^{(1)}=\left(\begin{array}{cc}m_{d} & \omega_{d} \\ X_{d}^{(1)} & M_{d}^{(1)}\end{array}\right), \quad \mathcal{M}_{u}^{(1)}=\left(\begin{array}{cc}m_{u} & \omega_{u} \\ X_{u}^{(1)} & M_{u}^{(1)}\end{array}\right)$
From this it is evident that the submatrix $R_{u}^{\dagger} K_{d}$ scales to $\rho_{u}^{-1} R_{u}^{\dagger} K_{d}$ and consequently we have a very simple scaling law from the up heavy sector scaling:

$$
\begin{aligned}
X_{u} & \rightarrow \rho_{u} X_{u} \quad, \quad M_{u} \rightarrow \rho_{u} M_{u}, \\
V_{U_{i} d_{j}} & \rightarrow \rho_{u}^{-1} V_{U_{i} d_{j}}, \lambda_{d_{i} d_{j}}^{U_{i}} \rightarrow \rho_{u}^{-2} \lambda_{d_{i} d_{j}}^{U_{i}}, \\
m_{U_{i}} & \rightarrow \rho_{u} m_{U_{i}} .
\end{aligned}
$$


This scaling is sufficient-if needed-to suppress all loop induced effects. For example, in the case of $\Delta F=$ 2 processes, the first correction in Eq. (40) scales as $\left(\lambda_{q q^{\prime}}^{U_{i}}\right)^{2} S\left(x_{U_{i}}\right) \sim\left(\lambda_{q q^{\prime}}^{U_{i}}\right)^{2} m_{U_{i}}^{2}$ and therefore it decreases as $\rho_{u}^{-2}$. The second correction scales as $\rho_{u}^{-2} \ln \rho_{u}$ according to the behaviour of the IL function $S\left(x_{U_{i}}, x_{t}\right)$. Behaviour similar to the latter one appears in the $\Delta F=1$ processes following the cancellations explained in Eq. (44).

To be more specific we present briefly the results for a second example with $\mathcal{M}_{u}^{(2)}$ constructed with $\rho_{u}=2$ and keeping the rest of the model unchanged. For $\Delta F=2$ processes we get, instead of the results in Eq. (43), the following:

$$
\left(\begin{array}{c}
\Delta\left(K^{0}\right) \\
\Delta\left(B_{d}^{0}\right) \\
\Delta\left(B_{s}^{0}\right)
\end{array}\right)=\left(\begin{array}{l}
0.01 \\
0.07 \\
0.06
\end{array}\right),\left(\begin{array}{c}
\delta \phi\left(K^{0}\right) \\
\delta \phi\left(B_{d}^{0}\right) \\
\delta \phi\left(B_{s}^{0}\right)
\end{array}\right)=\left(\begin{array}{l}
0.06 \\
0.01 \\
0.01
\end{array}\right)
$$

On average, the corrections to the SM mixing values get reduced from a $12 \%$ to some $6 \%$. In the case of $\Delta F=1$ processes instead of Eq. (45) we get

$$
\begin{aligned}
& r\left(K_{L} \rightarrow \mu \bar{\mu}\right)_{S D}=0.16, \quad r\left(B_{d} \rightarrow \mu \bar{\mu}\right)=0.16, \\
& r\left(B_{S} \rightarrow \mu \bar{\mu}\right)=0.16, \\
& r\left(K^{+} \rightarrow \pi^{+} \nu \bar{v}\right)=0.11, \quad r\left(B^{+} \rightarrow \pi^{+} \nu \bar{v}\right)=0.11, \\
& r\left(B^{+} \rightarrow K^{+} \nu \bar{v}\right)=0.10 .
\end{aligned}
$$

In this sector, the deviation from the SM model gets reduced by a factor of 2 . In this model "(2)" the deviations in these processes are at the 11-16\% level, showing a smooth decoupling of the effects while at the same time the heavy up-type quarks get heavier masses: $\left(m_{U_{1}}, m_{U_{2}}, m_{U 3}\right) \sim(2.6,3,4.5)$ $\mathrm{TeV}$. As for the oblique corrections, they have the following values:

$\Delta T=0.11, \Delta S=0.05, \Delta U=0.004$.

Finally for $\left(\left|V_{t d}\right|,\left|V_{t s}\right|,\left|V_{t b}\right|\right)$ we get $(0.008575,0.040258$, 0.99632 ), since $K_{u}$ deviates less from unitarity as a consequence of the reduction of $R_{u}^{\dagger} K_{d}$ by a factor of 2 .

We could also have scaled by a factor $\rho_{d}>1$, both $X_{d}^{(1)}$ and $M_{d}^{(1)}$, thus increasing the masses of all heavy quarks. This change has almost no effect at low energies since FCNC in the down sector are highly suppressed in $W^{d}$ and $\mathcal{H}_{\text {eff }}^{d}$ does not change at leading order.

\section{Conclusions}

We have presented a simple solution to a novel fine-tuning problem present in the SM. The solution involves the introduction of a $Z_{6}$ flavour symmetry, together with vector-like quarks of $Q=-1 / 3$ and $Q=2 / 3$ charges, as well as a complex singlet scalar. In the absence of vector-like quarks only the bottom and the top quarks acquire masses and $V_{\mathrm{CKM}}=\mathbb{1}$. We have shown that in the presence of the vector-like quarks which mix with the standard quarks, a realistic quark mass spectrum can be obtained and a correct CKM matrix can be generated. It is remarkable that these results are obtained in a framework where $Z_{6}$ is an exact symmetry of the Lagrangian, only softly broken in the scalar potential. This breaking is essential in order to avoid the domain-wall problem. In the literature, there are a large number of works addressing the flavour problem, [65-69] in various frameworks, including supersymmetric models or in scenarios including also mirror fermions or vector-like quarks. The distinctive feature of the present work is the fact that the full $V_{\mathrm{CKM}}$ matrix is entirely generated through the mixing with vector-like quarks. We have presented specific realistic examples and have analysed various FCNC processes as well as the decay channels of the vector-like quarks. It is also remarkable that in the framework of fully realistic models, some of these vector-like quarks are within the reach of the second LHC run.

Acknowledgements The authors acknowledge financial support from the Spanish MINECO under Grant FPA2015-68318-R, by the Severo Ochoa Excellence Center Project SEV-2014-0398, by Generalitat Valenciana under Grant PROMETEOII/ 2014/049 and by Fundação para a Ciência e a Tecnologia (FCT, Portugal) through the projects CERN/FIS-NUC/0010/2015, and CFTP-FCT Unit 777 (UID/FIS/00777 12013) which are partially funded through POCTI (FEDER), COMPETE, QREN and EU. M.N. acknowledges support from FCT through the postdoctoral Grant SFRH/BPD/112999/2015. The authors also acknowledge the hospitality of Universidad de Valencia, IFIC, and CFTP at IST Lisboa during visits for scientific collaboration.

Open Access This article is distributed under the terms of the Creative Commons Attribution 4.0 International License (http://creativecomm ons.org/licenses/by/4.0/), which permits unrestricted use, distribution, and reproduction in any medium, provided you give appropriate credit to the original author(s) and the source, provide a link to the Creative Commons license, and indicate if changes were made. Funded by SCOAP ${ }^{3}$.

\section{Appendix A: Derivation of effective Hermitian squared mass matrix}

In order to derive the expression given for $\mathcal{H}_{\text {eff }}$ in Eq. (24) we start from

$\mathcal{M} \mathcal{M}^{\dagger} \mathcal{U}_{L}=\mathcal{U}_{L}\left(\begin{array}{cc}d^{2} & o \\ 0 & D^{2}\end{array}\right)$

using the notation of Eq. (17), where, for simplicity, we have dropped the indices $d$ and $u$ for down and up. We can rewrite this equation explicitly, in terms of each one of the four $3 \times 3$ blocks, using Eq. (10) for the matrix $\mathcal{M}$ : 
$\left(m m^{\dagger}+\omega \omega^{\dagger}\right) K+\left(m X^{\dagger}+\omega M^{\dagger}\right) S=K d^{2}$,

$\left(m m^{\dagger}+\omega \omega^{\dagger}\right) R+\left(m X^{\dagger}+\omega M^{\dagger}\right) T=R D^{2}$,

$\left(X m^{\dagger}+M \omega^{\dagger}\right) K+\left(X X^{\dagger}+M M^{\dagger}\right) S=S d^{2}$,

$\left(X m^{\dagger}+M \omega^{\dagger}\right) R+\left(X X^{\dagger}+M M^{\dagger}\right) T=T D^{2}$.

Since the term $S d^{2}$ is much smaller than the other two, Eq. (63) can be approximated by

$\left(X X^{\dagger}+M M^{\dagger}\right) S \simeq-\left(X m^{\dagger}+M \omega^{\dagger}\right) K$

or else

$S \simeq-\left(X X^{\dagger}+M M^{\dagger}\right)^{-1}\left(X m^{\dagger}+M \omega^{\dagger}\right) K$

Replacing $S$ in Eq. (61) we obtain to a good approximation

$$
\begin{aligned}
& {\left[\left(m m^{\dagger}+\omega \omega^{\dagger}\right)-\left(m X^{\dagger}+\omega M^{\dagger}\right)\right.} \\
& \left.\quad \times\left(X X^{\dagger}+M M^{\dagger}\right)^{-1}\left(X m^{\dagger}+M \omega^{\dagger}\right)\right] K=K d^{2},
\end{aligned}
$$

implying

$K^{-1} \mathcal{H}_{\mathrm{eff}} K=d^{2}$

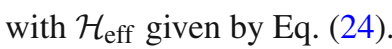

\section{Appendix B: Vector-like quark decays}

The functions that appear in Sect. 4.3 that are needed for the heavy quark decays are

$f_{h}(x, y)=(1+y-x) f(x, y)$,

$f_{V}(x, y)=\left(\left(1-y^{2}\right)+x(1+y)-2 x^{2}\right) f(x, y)$,

$V=Z, W^{ \pm}$

where

$f(x, y)=\sqrt{1-(\sqrt{y}+\sqrt{x})^{2}} \sqrt{1-(\sqrt{y}-\sqrt{x})^{2}}$.

Let us explain the origin and validity of the relations

$\left|V_{j i^{\prime}}\right|^{2} \sim\left|\left(W^{u}\right)_{i j}\right|^{2}, \quad\left|V_{i^{\prime} j}\right|^{2} \sim\left|\left(W^{d}\right)_{i j}\right|^{2}$,

where the prime means that $i$ and $i^{\prime}$ correspond to the same generation number, $i=i^{\prime}$. For that purpose we introduce, in self-explanatory matrix notation,

$V=\left(\begin{array}{cc}V_{u d} & V_{u D} \\ V_{U d} & V_{U D}\end{array}\right)$ where each submatrix connects the corresponding types of quarks $u, U$ and $d, D$ (in the present model, all four submatrices are $3 \times 3$ ). Similarly, we also introduce

$W^{u}=\left(\begin{array}{cc}W_{u u}^{u} & W_{u U}^{u} \\ W_{U u}^{u} & W_{U U}^{u}\end{array}\right), \quad W^{d}=\left(\begin{array}{cc}W_{d d}^{d} & W_{d D}^{d} \\ W_{D d}^{d} & W_{D D}^{d}\end{array}\right)$

Following again the notation in Sect. 3.3 for the up and down sectors, we have

$V_{U d}=R_{u}^{\dagger} K_{d}, \quad W_{U u}^{u}=R_{u}^{\dagger} K_{u}$,

so we can write

$V_{U d}=W_{U u}^{u} K_{u}^{-1} K_{d}$

At leading order $K_{u}^{-1} \sim K_{u}^{\dagger}$ and we get, to a high accuracy (and similarly for $V_{u D}$ and $W_{d D}^{d}$ )

$V_{U d} \sim W_{U u}^{u} V_{u d}, \quad V_{u D} \sim V_{u d} W_{d D}^{d}$

From these expressions it is easy to prove that the relations in Eq. (71) hold, at least for the dominant decay channel of the corresponding heavy up or down quark. The key ingredient is that $V_{u d} \sim \mathbb{I}$, in Eq. (72), with corrections at most of order $\lambda=0.22$.

\section{References}

1. K.A. Olive et al. [Particle Data Group Collaboration], Chin. Phys. C 38, 090001 (2014). doi:10.1088/1674-1137/38/9/090001

2. Y. Amhis et al. [Heavy Flavor Averaging Group (HFAG) Collaboration], Averages of $b$-hadron, $c$-hadron, and $\tau$-lepton properties as of summer (2014). arXiv: 1412.7515 [hep-ex]

3. F.C. Porter, Experimental status of the CKM matrix. Prog. Part. Nucl. Phys. 91, 101 (2016). doi:10.1016/j.ppnp.2016.06.003. arXiv: 1604.04940 [hep-ex]

4. F.J. Botella, G.C. Branco, M. Nebot, M.N. Rebelo, New physics and evidence for a complex CKM. Nucl. Phys. B 725, 155 (2005). doi:10.1016/j.nuclphysb.2005.07.006. arXiv:hep-ph/0502133

5. J. Charles et al. [CKMfitter Group Collaboration], CP violation and the CKM matrix: assessing the impact of the asymmetric $B$ factories. Eur. Phys. J. C 41(1), 1 (2005). doi:10.1140/epjc/ s2005-02169-1. arXiv:hep-ph/0406184

6. M. Bona et al. [UTfit Collaboration], The 2004 UTfit collaboration report on the status of the unitarity triangle in the standard model. JHEP 0507, 028 (2005). doi:10.1088/1126-6708/2005/07/ 028. arXiv:hep-ph/0501199

7. G. Aad et al. [ATLAS Collaboration], Observation of a new particle in the search for the Standard Model Higgs boson with the ATLAS detector at the LHC. Phys. Lett. B 716, 1 (2012). doi:10.1016/j. physletb.2012.08.020. arXiv:1207.7214 [hep-ex]

8. S. Chatrchyan et al. [CMS Collaboration], Observation of a new boson at a mass of $125 \mathrm{GeV}$ with the CMS experiment at the LHC. Phys. Lett. B 716, 30 (2012). doi:10.1016/j.physletb.2012.08.021. arXiv:1207.7235 [hep-ex] 
9. F.J. Botella, G.C. Branco, M.N. Rebelo, J.I. Silva-Marcos, What if the masses of the first two quark families are not generated by the standard model Higgs boson? Phys. Rev. D 94(11), 115031 (2016). doi:10.1103/PhysRevD.94.115031. arXiv:1602.08011 [hep-ph]

10. G. Aad et al. [ATLAS Collaboration], Search for pair production of a new heavy quark that decays into a $W$ boson and a light quark in $p p$ collisions at $\sqrt{s}=8 \mathrm{TeV}$ with the ATLAS detector. Phys. Rev. D 92(11), 112007 (2015). doi:10.1103/PhysRevD.92.112007. arXiv:1509.04261 [hep-ex]

11. G. Aad et al. [ATLAS Collaboration], Search for vector-like $B$ quarks in events with one isolated lepton, missing transverse momentum and jets at $\sqrt{s}=8 \mathrm{TeV}$ with the ATLAS detector. Phys. Rev. D 91(11), 112011 (2015). doi:10.1103/PhysRevD.91. 112011. arXiv:1503.05425 [hep-ex]

12. G. Aad et al. [ATLAS Collaboration], Search for production of vector-like quark pairs and of four top quarks in the lepton-plusjets final state in $p p$ collisions at $\sqrt{s}=8 \mathrm{TeV}$ with the ATLAS detector. JHEP 1508, 105 (2015). doi:10.1007/JHEP08(2015)105. arXiv:1505.04306 [hep-ex]

13. G. Aad et al. [ATLAS Collaboration], Search for the production of single vector-like and excited quarks in the $W t$ final state in $p p$ collisions at $\sqrt{s}=8 \mathrm{TeV}$ with the ATLAS detector. JHEP 1602, 110 (2016). doi:10.1007/JHEP02(2016)110. arXiv:1510.02664 [hepex]

14. G. Aad et al. [ATLAS Collaboration], Search for single production of vector-like quarks decaying into $\mathrm{Wb}$ in pp collisions at $\sqrt{s}=8 \mathrm{TeV}$ with the ATLAS detector. Eur. Phys. J. C 76(8), 442 (2016). doi:10.1140/epjc/s10052-016-4281-8. arXiv:1602.05606 [hep-ex]

15. G. Aad et al. [ATLAS Collaboration], Search for single production of a vector-like quark via a heavy gluon in the $4 b$ final state with the ATLAS detector in $p p$ collisions at $\sqrt{s}=8 \mathrm{TeV}$. Phys. Lett. B 758, 249 (2016). doi:10.1016/j.physletb.2016.04.061. arXiv:1602.06034 [hep-ex]

16. S. Chatrchyan et al. [CMS Collaboration], Inclusive search for a vector-like T quark with charge $\frac{2}{3}$ in pp collisions at $\sqrt{s}=8 \mathrm{TeV}$. Phys. Lett. B 729, 149 (2014). doi:10.1016/j.physletb.2014.01.006. arXiv:1311.7667 [hep-ex]

17. V. Khachatryan et al. [CMS Collaboration], Search for vector-like T quarks decaying to top quarks and Higgs bosons in the all-hadronic channel using jet substructure. JHEP 1506, 080 (2015). doi:10. 1007/JHEP06(2015)080. arXiv:1503.01952 [hep-ex]

18. V. Khachatryan et al. [CMS Collaboration], Search for pairproduced vectorlike B quarks in proton-proton collisions at $\sqrt{s}=8$ TeV. Phys. Rev. D 93(11), 112009 (2016). doi:10.1103/ PhysRevD.93.112009. arXiv:1507.07129 [hep-ex]

19. V. Khachatryan et al. [CMS Collaboration], Search for vector-like charge $2 / 3 \mathrm{~T}$ quarks in proton-proton collisions at $\sqrt{s}=8 \mathrm{TeV}$. Phys. Rev. D 93(1), 012003 (2016). doi:10.1103/PhysRevD.93. 012003. arXiv:1509.04177 [hep-ex]

20. F. del Aguila, M.K. Chase, J. Cortes, Vector-like fermion contributions to epsilon-prime. Nucl. Phys. B 271, 61 (1986). doi:10.1016/ S0550-3213(86)80004-9. doi:10.1016/0550-3213(86)90354-8

21. G.C. Branco, L. Lavoura, On the addition of vector like quarks to the standard model. Nucl. Phys. B 278, 738 (1986). doi:10.1016/ 0550-3213(86)90060-X

22. F. del Aguila, E. Laermann, P.M. Zerwas, Exotic E(6) particles in $e^{+} e^{-}$annihilation. Nucl. Phys. B 297, 1 (1988). doi:10.1016/ 0550-3213(88)90197-6

23. F. del Aguila, G.L. Kane, M. Quiros, Could the quark electroweak and mass eigenstates coincide? Phys. Lett. B 196, 531 (1987). doi:10.1016/0370-2693(87)90815-X

24. P. Langacker, D. London, Mixing between ordinary and exotic fermions. Phys. Rev. D 38, 886 (1988). doi:10.1103/PhysRevD. 38.886
25. F. del Aguila, L. Ametller, G.L. Kane, J. Vidal, Vector like fermion and standard Higgs production at hadron colliders. Nucl. Phys. B 334, 1 (1990). doi:10.1016/0550-3213(90)90655-W

26. L. Bento, G.C. Branco, P.A. Parada, A minimal model with natural suppression of strong CP violation. Phys. Lett. B 267, 95 (1991). doi:10.1016/0370-2693(91)90530-4

27. T.P. Cheng, L.F. Li, Suppression of flavor changing neutral current effects due to mixings with a heavy singlet fermion. Phys. Rev. D 45, 1708 (1992). doi:10.1103/PhysRevD.45.1708

28. L. Lavoura, J.P. Silva, Bounds on the mixing of the down type quarks with vector-like singlet quarks. Phys. Rev. D 47, 1117 (1993). doi:10.1103/PhysRevD.47.1117

29. G.C. Branco, T. Morozumi, P.A. Parada, M.N. Rebelo, CP asymmetries in $\mathrm{B} 0$ decays in the presence of flavor changing neutral currents. Phys. Rev. D 48, 1167 (1993). doi:10.1103/PhysRevD. 48.1167

30. V.D. Barger, M.S. Berger, R.J.N. Phillips, Quark singlets: implications and constraints. Phys. Rev. D 52, 1663 (1995). doi:10.1103/ PhysRevD.52.1663. arXiv:hep-ph/9503204

31. G. Barenboim, F.J. Botella, G.C. Branco, O. Vives, How sensitive to FCNC can B0 CP asymmetries be? Phys. Lett. B 422, 277 (1998). doi:10.1016/S0370-2693(97)01515-3. arXiv:hep-ph/9709369

32. G. Barenboim, F.J. Botella, O. Vives, Tree level FCNC in the $B$ system: from $\mathrm{CP}$ asymmetries to rare decays. Phys. Rev. D 64, 015007 (2001). doi:10.1103/PhysRevD.64.015007. arXiv:hep-ph/0012197

33. G. Barenboim, F.J. Botella, O. Vives, Constraining models with vector-like fermions from FCNC in $K$ and $B$ physics. Nucl. Phys. B 613, 285 (2001). doi:10.1016/S0550-3213(01)00390-X. arXiv:hep-ph/0105306

34. J.A. Aguilar-Saavedra, Effects of mixing with quark singlets. Phys. Rev. D 67, 035003 (2003). Erratum: [Phys. Rev. D 69 (2004) 099901] doi:10.1103/PhysRevD.69.099901. doi:10.1103/ PhysRevD.67.035003. arXiv:hep-ph/0210112

35. J.A. Aguilar-Saavedra, F.J. Botella, G.C. Branco, M. Nebot, The size of $\chi=\arg \left(-V(t s) V^{*}(t b) V^{*}(c s) V(c b)\right)$ and physics beyond the standard model. Nucl. Phys. B 706, 204 (2005). doi:10.1016/j. nuclphysb.2004.11.024. arXiv:hep-ph/0406151

36. F.J. Botella, G.C. Branco, M. Nebot, Small violations of unitarity, the phase in $B_{s}^{0}-\bar{B}_{s}^{O}$ and visible $t \rightarrow c Z$ decays at the LHC. Phys. Rev. D 79, 096009 (2009). doi:10.1103/PhysRevD. 79.096009. arXiv:0805.3995 [hep-ph]

37. K. Higuchi, K. Yamamoto, Flavor-changing interactions with singlet quarks and their implications for the LHC. Phys. Rev. D 81, 015009 (2010). doi:10.1103/PhysRevD.81.015009. arXiv:0911.1175 [hep-ph]

38. G. Cacciapaglia, A. Deandrea, D. Harada, Y. Okada, Bounds and decays of new heavy vector-like top partners. JHEP 1011, 159 (2010). doi:10.1007/JHEP11(2010)159. arXiv:1007.2933 [hep$\mathrm{ph}]$

39. F.J. Botella, G.C. Branco, M. Nebot, The hunt for new physics in the flavour sector with up vector-like quarks. JHEP 1212, 040 (2012). doi:10.1007/JHEP12(2012)040. arXiv:1207.4440 [hep-ph]

40. J.A. Aguilar-Saavedra, R. Benbrik, S. Heinemeyer, M. PerezVictoria, Handbook of vectorlike quarks: mixing and single production. Phys. Rev. D 88(9), 094010 (2013). doi:10.1103/PhysRevD. 88.094010. arXiv:1306.0572 [hep-ph]

41. M. Buchkremer, G. Cacciapaglia, A. Deandrea, L. Panizzi, Model Independent framework for searches of top partners. Nucl. Phys. B 876, 376 (2013). doi:10.1016/j.nuclphysb.2013.08.010. arXiv:1305.4172 [hep-ph]

42. S. Fajfer, A. Greljo, J.F. Kamenik, I. Mustac, Light Higgs and vector-like quarks without prejudice. JHEP 1307, 155 (2013). doi:10.1007/JHEP07(2013)155. arXiv:1304.4219 [hep-ph] 
43. S.A.R. Ellis, R.M. Godbole, S. Gopalakrishna, J.D. Wells, Survey of vector-like fermion extensions of the Standard Model and their phenomenological implications. JHEP 1409, 130 (2014). doi:10. 1007/JHEP09(2014)130. arXiv:1404.4398 [hep-ph]

44. A.K. Alok, S. Banerjee, D. Kumar, S.U. Sankar, D. London, New-physics signals of a model with a vector-singlet up-type quark. Phys. Rev. D 92, 013002 (2015). doi:10.1103/PhysRevD. 92.013002. arXiv:1504.00517 [hep-ph]

45. G. Cacciapaglia, A. Parolini, Light t Hooft top partners. Phys. Rev. D 93(7), 071701 (2016). doi:10.1103/PhysRevD.93.071701. arXiv:1511.05163 [hep-ph]

46. K. Ishiwata, Z. Ligeti, M.B. Wise, New vector-like fermions and flavor physics. JHEP 1510, 027 (2015). doi:10.1007/ JHEP10(2015)027. arXiv:1506.03484 [hep-ph]

47. C. Bobeth, A.J. Buras, A. Celis, M. Jung, Patterns of flavour violation in models with vector-like quarks. arXiv:1609.04783 [hep-ph]

48. M. Buchkremer, A. Schmidt, Long-lived heavy quarks : a review. Adv. High Energy Phys. 2013, 690254 (2013). doi:10.1155/2013/ 690254. arXiv: 1210.6369 [hep-ph]

49. R. Dermisek, Unification of gauge couplings in the standard model with extra vectorlike families. Phys. Rev. D 87(5), 055008 (2013). doi:10.1103/PhysRevD.87.055008. arXiv:1212.3035

50. C.D. Froggatt, H.B. Nielsen, Hierarchy of quark masses, Cabibbo angles and CP violation. Nucl. Phys. B 147, 277 (1979). doi:10. 1016/0550-3213(79)90316-X

51. Z.z Xing, H. Zhang, S. Zhou, Updated values of running quark and lepton masses. Phys. Rev. D 77, 113016 (2008). doi:10.1103/ PhysRevD.77.113016. arXiv:0712.1419 [hep-ph]

52. Z.Z Xing, H. Zhang, S. Zhou, Impacts of the Higgs mass on vacuum stability, running fermion masses and two-body Higgs decays. Phys. Rev. D 86, 013013 (2012). doi:10.1103/PhysRevD. 86.013013. arXiv: 1112.3112 [hep-ph]

53. G.C. Branco, L. Lavoura, J.P. Silva CP violation. Int. Ser. Monogr. Phys. 103, 1-536 (1999)

54. T. Inami, C.S. Lim, Effects of superheavy quarks and leptons in low-energy weak processes $K_{L} \rightarrow \mu \bar{\mu}, K^{+} \rightarrow \pi^{+} \nu \bar{\nu}$ and $K^{0} \leftrightarrow$ $\bar{K}^{0}$. Prog. Theor. Phys. 65, 297 (1981). Erratum: [Prog. Theor. Phys. 65 (1981) 1772]. doi:10.1143/PTP.65.297

55. G. Barenboim, F.J. Botella, Delta $F=2$ effective Lagrangian in theories with vector-like fermions. Phys. Lett. B 433, 385 (1998). doi:10.1016/S0370-2693(98)00695-9. arXiv:hep-ph/9708209

56. E. Nardi, Top-charm flavor changing contributions to the effective bsZ vertex. Phys. Lett. B 365, 327 (1996). doi:10.1016/ 0370-2693(95)01308-3. arXiv:hep-ph/9509233
57. M.I. Vysotsky, New (virtual) physics in the era of the LHC. Phys. Lett. B 644, 352 (2007). doi:10.1016/j.physletb.2006.12.003. arXiv:hep-ph/0610368

58. P.N. Kopnin, M.I. Vysotsky, Manifestation of a singlet heavy uptype quark in the branching ratios of rare decays $K \rightarrow \pi \nu \bar{v}, B \rightarrow$ $\pi \nu \bar{v}$ and $B \rightarrow K \nu \bar{v}$. JETP Lett. 87, 517 (2008). doi:10.1134/ S0021364008100019. arXiv:0804.0912 [hep-ph]

59. I. Picek, B. Radovcic, Nondecoupling of terascale isosinglet quark and rare K- and B-decays. Phys. Rev. D 78, 015014 (2008). doi: 10. 1103/PhysRevD.78.015014. arXiv:0804.2216 [hep-ph]

60. C. Bobeth, M. Gorbahn, T. Hermann, M. Misiak, E. Stamou, M. Steinhauser, $B_{s, d} \rightarrow l^{+} l^{-}$in the standard model with reduced theoretical uncertainty. Phys. Rev. Lett. 112, 101801 (2014). doi:10. 1103/PhysRevLett.112.101801. arXiv:1311.0903 [hep-ph]

61. V. Khachatryan et al. [CMS and LHCb Collaborations], Observation of the rare $B_{s}^{0} \rightarrow \mu^{+} \mu^{-}$decay from the combined analysis of CMS and LHCb data. Nature 522, 68 (2015). doi:10.1038/ nature14474. arXiv:1411.4413 [hep-ex]

62. S. Descotes-Genon, L. Hofer, J. Matias, J. Virto, Global analysis of $b \rightarrow$ sll anomalies. JHEP 1606, 092 (2016). doi:10.1007/ JHEP06(2016)092. arXiv:1510.04239 [hep-ph]

63. M.E. Peskin, T. Takeuchi, Estimation of oblique electroweak corrections. Phys. Rev. D 46, 381 (1992). doi:10.1103/PhysRevD.46. 381

64. L. Lavoura, J.P. Silva, The Oblique corrections from vector-like singlet and doublet quarks. Phys. Rev. D 47, 2046 (1993). doi:10. 1103/PhysRevD.47.2046

65. M. Leurer, Y. Nir, N. Seiberg, Mass matrix models. Nucl. Phys. B 398, 319 (1993). doi:10.1016/0550-3213(93)90112-3. arXiv:hep-ph/9212278

66. A. Pomarol, D. Tommasini, Horizontal symmetries for the supersymmetric flavor problem. Nucl. Phys. B 466, 3 (1996). doi:10. 1016/0550-3213(96)00074-0. arXiv:hep-ph/9507462

67. O. Gedalia, G. Perez, Flavor physics. doi:10.1142/ 9789814327183_0006. arXiv:1005.3106 [hep-ph]

68. R. Barbieri, G. Isidori, J. Jones-Perez, P. Lodone, D.M. Straub, $U(2)$ and minimal flavour violation in supersymmetry. Eur. Phys. J. C 71, 1725 (2011). doi:10.1140/epjc/s10052-011-1725-z. arXiv:1105.2296 [hep-ph]

69. A.J. Buras, C. Grojean, S. Pokorski, R. Ziegler, FCNC effects in a minimal theory of fermion masses. JHEP 1108, 028 (2011). doi: 10. 1007/JHEP08(2011)028. arXiv:1105.3725 [hep-ph] 